\title{
Newly detected liver nodules with a history of colorectal cancer: are they metastatic? Review of 2,632 cases in a single center
}

\author{
Kai Zhu ${ }^{1,2 \#}$, Wentao Wang ${ }^{3 \#}$, Rongkui Luo ${ }^{4 \#}$, Danjun Song ${ }^{1}$, Xi Wang ${ }^{5}$, Qiang Gao ${ }^{1}$, Jia Fan ${ }^{1,2,6,7}$, \\ Jian Zhou ${ }^{1,2,6,7}$, Shengxiang Rao ${ }^{2}$, Xiaoying Wang ${ }^{1}$ \\ ${ }^{1}$ Department of Liver Surgery and Transplantation, Liver Cancer Institute, Zhongshan Hospital, Fudan University; Key Laboratory of \\ Carcinogenesis and Cancer Invasion (Fudan University), Ministry of Education, Shanghai, China; ${ }^{2}$ Key Laboratory of Organ Transplantation, \\ Shanghai, China; ${ }^{3}$ Department of Radiology, Zhongshan Hospital, Fudan University; Shanghai Institute of Medical Imaging, Shanghai, China; \\ ${ }^{4}$ Department of Pathology, Zhong Shan Hospital, Fudan University, Shanghai, China; ${ }^{5}$ Department of Ultrasound, Zhong Shan Hospital, Fudan \\ University, Shanghai, China; ${ }^{6}$ Key Laboratory of Medical Epigenetics and Metabolism, Institute of Biomedical Sciences, Fudan University, Shanghai, \\ China; ${ }^{7}$ State Key Laboratory of Genetic Engineering, Fudan University, Shanghai, China \\ Contributions: (I) Conception and design: K Zhu, X Wang; (II) Administrative support: X Wang; (III) Provision of study materials or patients: R Luo; \\ (IV) Collection and assembly of data: D Song; (V) Data analysis and interpretation: K Zhu, W Wang, R Luo; (VI) Manuscript writing: All authors; (VII) \\ Final approval of manuscript: All authors. \\ "These authors contributed equally to this work. \\ Correspondence to: Xiaoying Wang. Department of Liver Surgery, Liver Cancer Institute, Zhongshan Hospital, Fudan University; Key Laboratory of \\ Carcinogenesis and Cancer Invasion of Ministry of Education, Shanghai 200032, China. Email: xiaoyingwang@fudan.edu.cn.
}

Background: The diagnosis of newly detected liver nodules in patients with colorectal cancer (CRC) is crucial for determining prognosis and treatment. Accurate identification of benign nodules can help avoid unnecessary therapy. The aim of our study was to retrospectively review patients with CRC who underwent liver resection for benign liver nodules misdiagnosed as CRC metastasis (CRLM) in our institution.

Methods: We reviewed all patients with a history of CRC who underwent liver resection from January 2012 to December 2019 in our institution. We specifically focused on nodules pathologically confirmed as benign. The pathology was rechecked by an independent pathologist. The clinicopathological characteristics of these patients were collected. Preoperative imaging examinations, including ultrasound (US), magnetic resonance imaging $(\mathrm{MRI})$, and positron emission tomography-computed tomography (PET-CT) were reviewed.

Results: From 2012 to 2019, a total of 2,632 patients with CRC who were preoperatively diagnosed CRLM received liver resection, among which 2,584 (98.2\%) cases were proven to be malignant, and 48 (1.8\%) cases were benign. Among these 48 cases, 24 were pathologically confirmed as focal nodular hyperplasia (FNH), 9 were peliosis, 10 were inflammatory lesions, and 5 were hemangioma. At least one preoperative imaging examination indicated CRLM, with a median size of $2.0 \mathrm{~cm}$ (range, $0.4-8.0 \mathrm{~cm}$ ). Before liver resection, ten patients received chemotherapy after the discovery of liver nodules.

Conclusions: It should be noted that newly detected liver nodules in patients with a history of CRC could be benign. Accurate diagnosis of liver nodules in CRC is necessary to avoid overtreatment and to identify cost-effective medication.

Keywords: Colorectal cancer liver metastasis (CRLM); focal nodular hyperplasia (FNH); peliosis hepatis; hemangioma

Submitted Dec 24, 2020. Accepted for publication Apr 05, 2021.

doi: $10.21037 / \mathrm{atm}-20-8153$

View this article at: http://dx.doi.org/10.21037/atm-20-8153 


\section{Introduction}

Colorectal cancer (CRC) is one of the most common malignancies worldwide. According to GLOBOCAN 2018, over 1.8 million new CRC cases and 881,000 deaths occurred in 2018, ranking third in terms of incidence but second in terms of mortality (1). The liver is the most common site for CRC metastasis (CRLM), which is the most common cause of death. Over $50 \%$ of patients with CRC will eventually develop liver metastasis (2), which needs immediate interference (3).

Surgical resection remains the most effective treatment for CRLM (4-6). Studies have shown that selected patients undergoing surgery to remove CRLM can have a median 5 -year survival of $38 \%$ (7); for patients with solitary liver metastases, the 5 -year overall survival rate can reach as high as $71 \%$ following resection $(8,9)$. Therefore, frequent surveillance and early diagnosis of liver metastasis are crucial for patients with CRC (3). The National Comprehensive Cancer Network (NCCN) suggests that patients with CRC at high risk of recurrence or metastasis should undergo abdominal CT every 3 to 6 months for 2 years, and then every 6 to 12 months for up to 5 years (10).

The incidence of benign liver lesions is very high in patients with CRC (11-14). Schwartz et al. (14) reviewed CT images obtained from 435 patients with CRC during a 24-month period, and found small hepatic lesions (lesions $1 \mathrm{~cm}$ or less in diameter) in $13 \%(\mathrm{n}=57)$ of the patients, among which only $14 \%(8 / 57)$ were metastases. Jang et al. (11) found that small liver nodules (15 $\mathrm{mm}$ or smaller) existed in $25.5 \%$ of patients with CRC, among which only $11.2 \%$ were metastases. CRLM could share similar imaging features with benign liver nodules on imaging examinations, including ultrasound (US), magnetic resonance imaging (MRI), and positron emission tomography-computed tomography (PET-CT), especially for small ones $(20 \mathrm{~mm}$ or smaller) $(15,16)$. The actual number of small hepatic lesions in patients with CRC was certainly underreported, which highlights the importance of accurate diagnosis of indeterminate liver nodules.

In our practice, we also noted that some newly detected liver nodules were radiologically misdiagnosed as CRLM and pathologically confirmed as benign. To analyze the reason for misdiagnosis, and to avoid further unnecessary surgeries and other invasive treatments, we retrospectively reviewed all patients with a history of CRC who underwent liver resection for liver nodules in our institution.

We present the following article in accordance with the
STROBE reporting checklist (available at http://dx.doi. org/10.21037/atm-20-8153),

\section{Methods}

The study was conducted in accordance with the Declaration of Helsinki (as revised in 2013). Our Institutional Review Board approved the retrospective study (B2021-229). Due to the nature of retrospective study, no written informed consent was obtained from patients.

\section{Study population}

We retrospectively reviewed all patients with CRC who underwent liver resection for liver nodules from January 2012 to December 2019 at Zhongshan Hospital of Fudan University. All the nodules were indicated to be CRLM by at least one imaging examination, and the indications for surgery were as follows (2): (I) based on liver anatomy and the extent of liver metastases, the metastatic lesions can be completely removed (R0) while preserving adequate liver function; (II) patients should be fit to undergo such surgical treatment, without extrahepatic metastases that are not suitable for surgery, or with only pulmonary nodules that do not restrict the resectability of the liver metastases. The diagnosis was confirmed by pathology, and those confirmed to be benign nodules were rechecked by an independent pathologist.

\section{Data collection}

Clinical information such as age, sex, preoperative carcinoembryonic antigen (CEA), tumor characteristics (diameter, number, location, etc.), and therapeutic approaches were collected. The detailed clinicopathological features are listed in Table 1. Preoperative imaging examinations, including US, MRI, and PET-CT were reviewed.

\section{Statistical analysis}

All data were collected retrospectively from the hospital's electronic database. Statistical analyses were performed with SPSS 17.0 software (SPSS, Chicago, IL).

\section{Results}

From 2012 to 2019, a total of 2,632 patients with CRC 
Table 1 Clinicopathological characteristics of the 48 patients

\begin{tabular}{|c|c|c|c|c|c|}
\hline Variables & $\mathrm{FNH}(\mathrm{N}=24)$ & Peliosis (N=9) & Inflammatory lesion $(\mathrm{N}=10)$ & Hemangioma $(\mathrm{N}=5)$ & Total $(\mathrm{N}=48)$ \\
\hline \multicolumn{6}{|l|}{ Sex, n (\%) } \\
\hline Male & $15(62.5 .0)$ & $4(44.4)$ & $5(50.0)$ & $3(75.0)$ & $27(56.3)$ \\
\hline Female & $9(37.5 .0)$ & $5(55.6)$ & $5(50.0)$ & $2(25.0)$ & $21(43.7)$ \\
\hline Single & $18(75.0)$ & $7(77.8)$ & $9(90.0)$ & $4(80.0)$ & $38(79.2)$ \\
\hline Multiple & $6(25.0)$ & $2(22.2)$ & $1(10.0)$ & $1(20.0)$ & $10(20.8)$ \\
\hline Tumor diameter $(\mathrm{cm})$, median (range) & $1.6(1.0$ to 4.8$)$ & 1.5 (1.0 to 7.0$)$ & 1.7 (0.4 to 5.0$)$ & $2.0(1.0$ to 8.0$)$ & $2.0(0.4$ to 8.0$)$ \\
\hline \multicolumn{6}{|l|}{ Tumor diameter (cm), n (\%) } \\
\hline \multicolumn{6}{|l|}{ Localization, n (\%) } \\
\hline Unilobar & $20(83.3)$ & $9(100.0)$ & $10(100.0)$ & $4(80.0)$ & $43(89.6)$ \\
\hline Bilobar & $4(16.7)$ & $0(0.0)$ & $0(0.0)$ & $1(20.0)$ & $5(10.4)$ \\
\hline \multicolumn{6}{|l|}{ Primary tumor, n (\%) } \\
\hline Colon & $14(58.3)$ & $3(33.3)$ & $7(70.0)$ & $4(80.0)$ & $28(58.3)$ \\
\hline Rectum & $10(41.7)$ & $6(66.7)$ & $3(30.0)$ & $1(20.0)$ & $20(41.7)$ \\
\hline \multicolumn{6}{|l|}{ History of hepatic metastases, n (\%) } \\
\hline No & $22(91.7)$ & $8(88.9)$ & $8(80.0)$ & $5(100.0)$ & $43(89.6)$ \\
\hline \multicolumn{6}{|l|}{ Chemotherapy for liver nodule, n (\%) } \\
\hline No & $18(75.0)$ & $8(88.9)$ & $9(90.0)$ & $3(60.0)$ & $38(79.2)$ \\
\hline Yes & $6(25.0)$ & $1(11.1)$ & $1(10.0)$ & $2(40.0)$ & $10(20.8)$ \\
\hline Misdiagnosed by US, n (\%) & $21 / 24(87.5)$ & $7 / 9(77.8)$ & $9 / 10(90.0)$ & $3 / 5(60.0)$ & 40/48 (83.3) \\
\hline Misdiagnosed by MRI, n (\%) & 17/24 (70.8) & $7 / 9(77.8)$ & $8 / 10(80.0)$ & $4 / 5(80.0)$ & $36 / 48(75.0)$ \\
\hline Misdiagnosed by PET-CT, n (\%) & $7 / 13(53.8)$ & 1/3 (33.3) & $3 / 4(75.0)$ & 2/3 (66.7) & $13 / 23(56.5)$ \\
\hline \multicolumn{6}{|c|}{ Number of false diagnosis (US, MRI, or PET-CT), n (\%) } \\
\hline 0 & $0(0.0)$ & $0(0.0)$ & $0(0.0)$ & $0(0.0)$ & $0(0.0)$ \\
\hline 1 & $7(29.2)$ & $4(44.4)$ & $2(20.0)$ & $2(40.0)$ & $15(31.3)$ \\
\hline 2 & $14(58.3)$ & $4(44.4)$ & $5(50.0)$ & $2(40.0)$ & $25(52.1)$ \\
\hline 3 & $3(12.5)$ & $1(11.1)$ & $3(30.0)$ & $1(20.0)$ & $8(16.7)$ \\
\hline
\end{tabular}

MRI, magnetic resonance imaging; US, ultrasonography; PET-CT, positron emission tomography-computed tomography. 


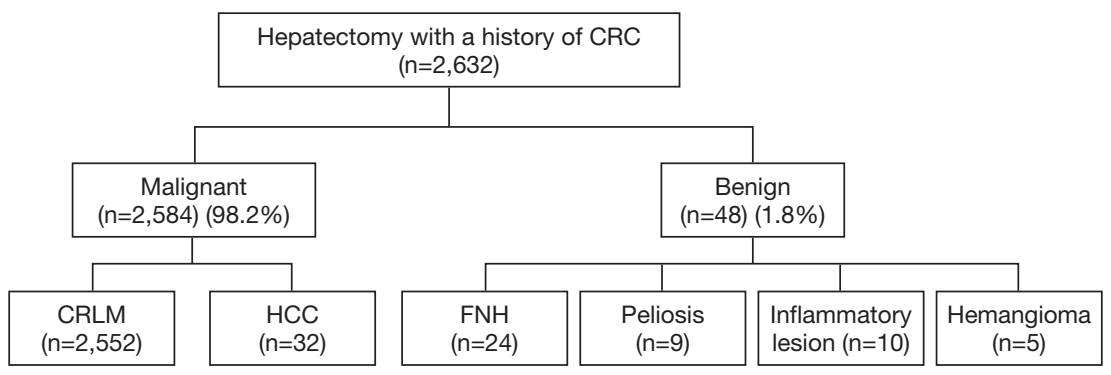

Figure 1 Study design and the number in each category.

who were preoperatively diagnosed CRLM received liver resection, and 2,584 cases were malignant (98.2\%), consisting of 2,552 CRLM and 32 HCC. The remaining 48 cases $(1.8 \%)$ were proven to be benign, among which 24 cases were diagnosed as FNH, 9 cases were hepatic peliosis, 10 cases were inflammatory lesions, and 5 cases were confirmed to be hemangioma (Figure 1).

The median age of the 48 patients was 59 years (range, 30-74 years), with 27 male and 21 female patients. Five of the 48 cases $(10.4 \%)$ had a prior history of liver surgery for hepatic metastasis; 39 of the 48 patients $(81.2 \%)$ had received chemotherapy (oxaliplatin, fluorouracil, or irinotecan) before the discovery of the liver nodules, and 10 patients $(20.8 \%)$ received chemotherapy after. The median size of the tumor was $2.0 \mathrm{~cm}$ (range, $0.4-8.0 \mathrm{~cm}$ ), with 36 (75\%) lesions less than $2 \mathrm{~cm}$. In 38 of the 48 cases (79.2\%), the lesion was single, and multiple lesions (two or more nodules) were observed in 10 cases (20.8\%) (Table 1). In these 48 patients, CEA levels were all normal. For all 48 patients, recovery was uneventful.

All 48 patients received US and MRI tests, and 23 patients received PET-CT tests. At least one preoperative imaging examination (US, MRI, or PET-CT) indicated CRLM, 15 cases had 1 false misdiagnosis, 25 had 2 false results, and 8 had 3 false results. Among these 3 imaging examinations, US had the highest rate of misdiagnosis [40/48 (83.3\%)], the misdiagnosis rate of MRI was 75\% (36/48), and PETCT had the lowest rate of misdiagnosis [13/23 (56.5\%)]. All 48 patients received preoperative color Doppler US, but none of them underwent contrast enhanced US for preoperative diagnosis. Likewise, all 48 patients received preoperative dynamic contrast-enhanced MRI with gadolinium, but none of them underwent gadoxetic acidenhanced MRI.

Most patients could not provide former liver imaging examinations, which we could compare with; therefore, we could not determine if the nodules had existed before
CRC or emerged after CRC. However, we also listed specific features of the nodules (CRLM, FNH, peliosis, inflammatory lesion, hemangioma) in the research.

\section{$C R L M$}

The appearance of CRLM on US is usually inhomogeneous. Virtually any sonographic appearance may occur in CRLM, and hypoechoic halos are common. The MR imaging features of most CRLMs were hypointense on T1-weighted imaging, mild-moderate hyperintense on T2-weighted imaging, hyperenhancement on arterial phase (AP), and isoor hyperintense on portal venous phase (PVP) (Figures 2,3).

\section{FNH}

FNHs generally have a capsule-free, well-circumscribed isoechoic appearance on US and may exhibit a "central scar". Doppler US usually shows the central arteries having a spokewheel pattern. The MR imaging features of most FNHs were hypointense on T1-weighted imaging, mild-moderate hyperintense on $\mathrm{T} 2$-weighted imaging, hyperenhancement on arterial phase (AP), and iso- or hyperintense on portal venous phase (PVP) (Figure 2). The median size of the FNH was $1.6 \mathrm{~cm}$ (range, $1.0-4.8 \mathrm{~cm}$ ). Tumor growth was observed in 2 of the 24 patients $(8.3 \%)$, where one nodule had grown from 0.5 to $2.3 \mathrm{~cm}$, and another from 0.5 to $1.5 \mathrm{~cm}$. All patients had received chemotherapy before the discovery of the liver nodules, and 6 (25\%) received chemotherapy after.

\section{Peliosis}

On US, peliosis may appear as pseudocystic areas in the hepatic parenchyma. These lesions can be hypoechoic in an otherwise normal liver, or they may appear hyperechoic in the setting of hepatic steatosis. Doppler studies can demonstrate the vascular nature of the lesion. The MR 

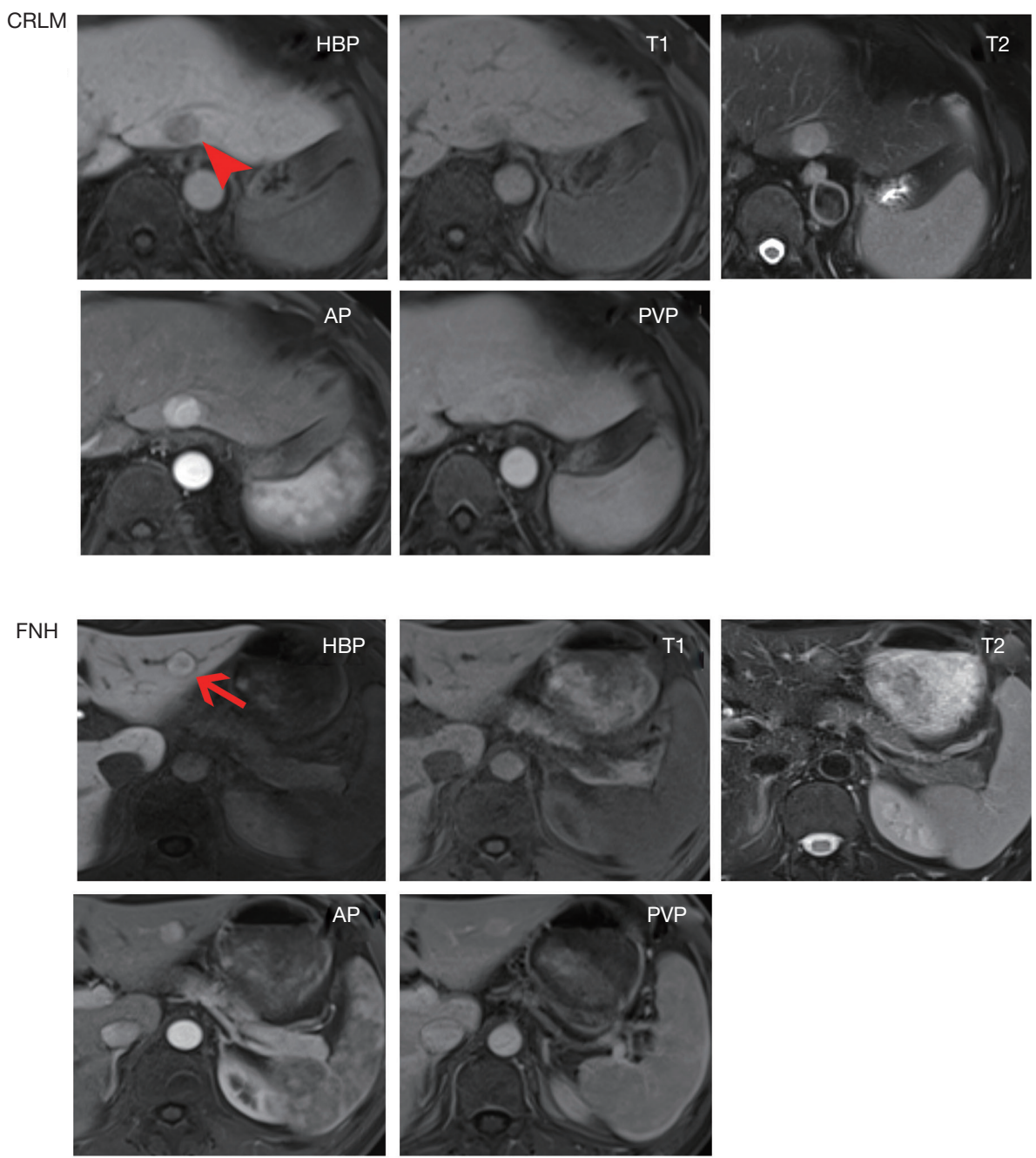

Figure 2 CRLM (red arrowhead) and FNH (red arrow) can be differentiated according to the signal intensity of HBP on gadoxetic acidenhanced MRI. CRLM: hypointensity on HBP, hypointensity on T1-weighted imaging, mild-moderate hyperintensity on T2-weighted imaging, hyperenhancement on AP, iso-or hyperintensity on PVP. FNH: hyperintensity on HBP, hypointensity on T1-weighted imaging, mild-moderate hyperintensity on T2-weighted imaging, hyperenhancement on AP, iso-or hyperintensity on PVP. HBP, hepatobiliary phase; AP, arterial phase; PVP, portal venous phase.

imaging features of peliosis are hypointense on T1weighted imaging, mild-moderate hyperintense on T2weighted imaging, mild enhancement on AP, and gradual enhancement on PVP (Figure 3). The median size was $1.5 \mathrm{~cm}$ (range, 1.0-7.0 cm). All patients had received chemotherapy before the discovery of the liver nodules, and $1(11.1 \%)$ received chemotherapy after.

\section{Inflammatory lesion}

The appearance of inflammatory lesion on US varies depending on the cause of the disease. The MR imaging features of most inflammatory lesions were hypointense on T1-weighted imaging, mild-moderate mild hyperintense on T2-weighted imaging, mild or peripheral enhancement on AP, and gradual enhancement on PVP (Figure 3). The median size was $1.7 \mathrm{~cm}$ (range, $0.4-5.0 \mathrm{~cm}$ ). Two cases were confirmed to be parasitosis, 1 case was an immunoglobulin G4 (IgG4)-related inflammatory lesion, and 1 patient had abdominal infection during previous surgery for colon cancer.

\section{Hemangioma}

Hemangioma is a well-circumscribed, round shaped hyper- 

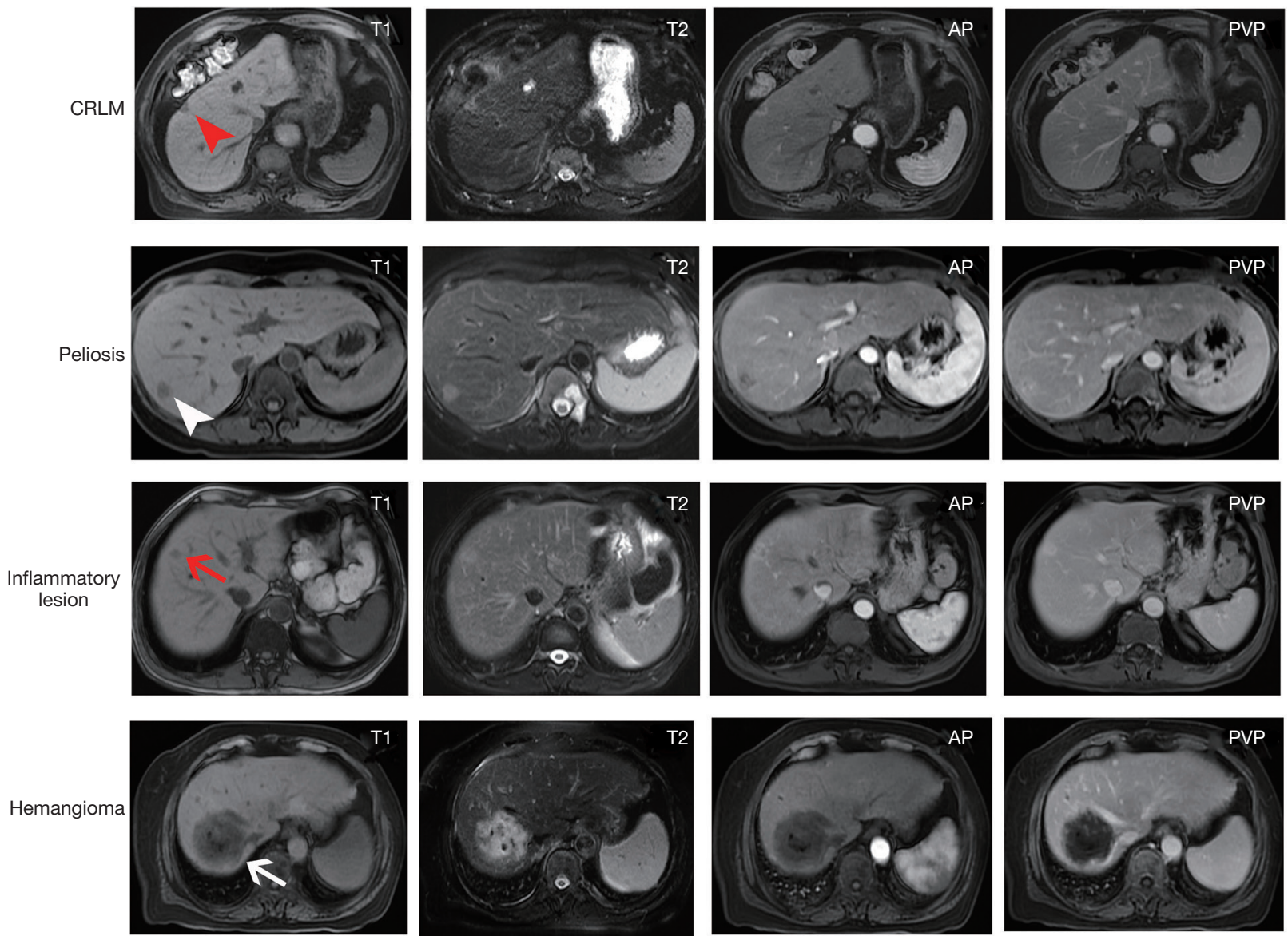

Figure 3 CRLM (red arrowhead), peliosis (white arrowhead), inflammatory lesion (red arrow), and hemangioma (white arrow) could share similar imaging features on dynamic contrast-enhanced MRI with gadolinium. CRLM: hypointensity on T1-weighted imaging, mildmoderate hyperintensity on T2-weighted imaging, peripheral enhancement on AP, mild or peripheral enhancement on PVP. Peliosis: hypointensity on T1-weighted imaging, mild-moderate hyperintensity on T2-weighted imaging, mild enhancement on AP, gradual enhancement on PVP. Inflammatory lesion: hypointensity on T1-weighted imaging, mild-moderate mild hyperintensity on T2-weighted imaging, mild or peripheral enhancement on AP, gradual enhancement on PVP. Atypical hemangioma: hypointensity on T1-weighted imaging, most moderate hyperintensity with intralesional low intensity on T2-weighted imaging, peripheral enhancement on AP, mild gradual enhancement on PVP. HBP, hepatobiliary phase; AP, arterial phase; PVP, portal venous phase.

echoic and homogeneous lesion on US. The MR imaging features of most hemangiomas can be hypointense on T1-weighted imaging, hyperintense on T2-weighted imaging, peripheral enhancement on AP, and mild gradual enhancement on PVP. The median size was $2.0 \mathrm{~cm}$ (range, $1.0-8.0 \mathrm{~cm})$, with $3(60 \%)$ lesions being less than $2 \mathrm{~cm}$. The $8 \mathrm{~cm}$ hemangioma cannot be easily diagnosed on MRI because the mass showed moderate hyperintensity rather than significant hyperintensity on T2-weighted imaging, due to the presence of fibrosis within the hemangioma (Figure 3).

\section{Discussion}

The diagnosis of newly discovered liver nodules in the background of CRC is important; however, the nodules are usually small, and differentiation between malignant and benign liver nodules may be challenging $(14,17)$. In this study, we reported 48 patients with CRC who underwent liver resection for benign liver nodules misdiagnosed as CRLM preoperatively in our institution from 2012 to 2019. These nodules were pathologically confirmed as benignities, including FNH, peliosis, inflammatory lesions, 
and hemangioma.

Although newly detected liver nodules in patients with CRC should be highly suggestive of metastasis, some benign nodules, such as focal nodular hyperplasia (FNH), hepatic peliosis, and steatosis, may occur in patients with CRC, especially after chemotherapy and molecularly targeted therapy (18). Accurate identification of liver nodules with a history of CRC can help avoid unnecessary therapy. Therefore, the diagnosis of small hepatic nodules in the background of CRC is important, but sometimes difficult.

In all 48 patients we reviewed, at least one preoperative imaging examination indicated CRLM. It is often difficult to characterize small indeterminate liver nodules with a history of CRC. For nodules inclined to be benign, fine needle aspiration is often useful to confirm the diagnosis. Nevertheless, pathological confirmation is not always essential if reasonable results can be achieved with imaging studies; in addition, it may cause potential dissemination for nodules to be malignant. PET-CT provides high accuracy in detecting local recurrence and distant metastasis, such as CRLM (19). However, false negative results are often seen in patients with CRLM, which might be due to negative uptake of fludeoxyglucose after receiving chemotherapy or targeted therapy.

In this study, we found that conventional Doppler US had the highest rate of misdiagnosis (83.3\%), partly because the result relies more on the subjective judgment of the examiner. In addition, it is challenging for grey-scale US to distinguish benign from malignant lesion $2 \mathrm{~cm}$ or less in diameter. Contrast-enhanced ultrasound (CEUS), on the contrary, can be used to observe the contrast agent in the lesions in real time, and is invaluable in providing characterization of indeterminate liver lesions on CT, MRI, and PET-CT (20). CEUS markedly improves the diagnostic accuracy of CRLM (21), FNH (22), hemangioma (23), etc. In addition, contrast agents, which are gas-filled particles (microbubbles), can be safely administered more than once during the same examination, and can be administered to pregnant women (24), patients with renal insufficiency (25), and without iodine/gadolinium-related anaphylactoidtype reaction (26). For these reasons, CEUS plays a complementary problem-solving role for indeterminate liver lesions

CRLM could share similar imaging features with $\mathrm{FNH}$, peliosis, inflammatory lesion, and hemangioma on dynamic contrast-enhanced MRI (Figures 2,3). FNH is the most common type to be misinterpreted as CRLM in this study.
It can be well characterized by a central stellate scar on contrast dynamic CT/MRI. However, the prevalence of the central scars is less than $50 \%$, and it is even lower when the lesion is smaller than $2 \mathrm{~cm}(15,16)$. Peliosis is also benign and characterized by a proliferation of the sinusoidal hepatic capillaries and cystic blood-filled cavities distributed in the liver (27). Studies have shown that chemotherapy-induced peliosis will regress after drug withdrawal, and no surgery is needed $(28,29)$. Studies also showed that peliosis could be misdiagnosed $(30,31)$.

In this study, we found that all patients with $\mathrm{FNH}$ and peliosis had received chemotherapy before the discovery of liver nodules. Chemotherapy has been shown to be associated with benign hepatic lesions, such as sinusoidal obstruction syndrome (SOS), FNH, and peliosis (18). SOS has been widely observed in patients receiving oxaliplatinbased chemotherapy $(18,32,33)$, which is characterized by occlusion of the terminal hepatic venules and hepatic sinusoids, as well as sinusoidal endothelial injury. Wicherts et al. (18) showed that SOS was observed in $15 \%$ of patients treated with oxaliplatin compared to $4 \%$ of patients treated with an oxaliplatin-naïve regimen. Several studies have demonstrated that $\mathrm{FNH}$ can be induced by sinusoidal obstruction syndrome (SOS) (34-36), and peliosis is also associated with SOS $(37,38)$. In addition, these lesions due to chemo injury could be misinterpreted as metastasis $(36,37,39)$, especially when the nodules are smaller than $1 \mathrm{~cm}$ in diameter $(14,17)$. In this study, the nodules were relatively small $(1.6 \mathrm{~cm}$ for $\mathrm{FNH}$ and $1.5 \mathrm{~cm}$ for peliosis), which increased the difficulty of accurate diagnosis.

In the inflammatory group, we observed various causes such as parasitosis, IgG4-related inflammatory lesions, and abdominal infection. The nodules showed various enhancement patterns on contrast-enhanced MRI, depending on the histopathologic findings, which makes the diagnosis much more complicated. Hemangioma is relatively easier to identify due to the typical appearance on MRI, which is a smooth, well-demarcated, homogeneous mass that has low signal intensity on T1-weighted images, hyperintense on $\mathrm{T} 2$-weighted images, and peripheral and gradual enhancement on contrast-enhanced imaging (40). However, in clinical practice, small liver nodules after chemotherapy may not demonstrate characteristic radiological features and cannot be diagnosed easily. In addition, giant hemangiomas may develop a collagenous scar or fibrous nodule, which makes the diagnosis more confusing (Figure 3) (41).

Gadoxetic acid-enhanced MRI is highly sensitive for 
differentiation between benign and malignant lesions, according to the signal intensity on the hepatobiliary phase (HBP) (42-44). On HBP, FNHs, peliosis, and some inflammatory lesions can be isointense or hyperintense (45-47), while CRLMs are usually hypointense (Figure 2) (48). The European Society for Medical Oncology (ESMO) suggests that for lesions $<10 \mathrm{~mm}$ in diameter, MRI is a more sensitive modality than CT, and gadoxetic acid-enhanced MRI is associated with higher accuracy of lesion detection (49-51). The China CRLM Guideline Group also recommends gadoxetic acid-enhanced MRI rather than conventional MRI or CT when necessary (2). In China, however, even hepatobiliary doctors have not fully realized the importance of gadoxetic acid-enhanced MRI, and due to the longer scan time and higher cost compared to dynamic contrast-enhanced MRI with gadolinium, gadoxetic acid-enhanced MRI has not been applied as a routine examination of liver imaging. In all 48 patients reviewed in our study, no one had performed gadoxetic acid-enhanced MRI. We also surveilled another 5 patients with CRC with suspected FNH who did not receive surgery or chemotherapy. All 5 patients underwent gadoxetic acidenhanced MRI, FNH was considered without pathological confirmation, and the liver nodules were stable to the latest follow-up. Therefore, for patients with CRC with small indeterminate liver nodules, we strongly recommend gadoxetic acid-enhanced MRI.

Our study has several limitations. Because of the retrospective, single-centered nature of the study, selection bias exists, and the true incidence of benign and malignant liver nodules in patients with CRC is surely underestimated. Large, multicenter, prospective studies are needed to increase the accuracy in identifying indeterminate liver nodules in patients with CRC.

\section{Conclusions}

Most patients with CRC would refer to surgeons when liver nodules first appear. With improvements in safety in hepatic surgery, overtreatment of liver nodules in patients with $\mathrm{CRC}$ is becoming increasingly intense. However, surgeries are always accompanied by potential morbidity and mortality, especially for patients with CRLM who receive preoperative chemotherapy. Since CEUS and gadoxetic acid-enhanced MRI can easily differentiate FNHs, peliosis, and some inflammatory lesions from CRLM, it should be offered to patients with CRC with indeterminate liver nodules, which could further minimize unnecessary surgeries and other invasive treatments.

\section{Acknowledgments}

Funding: This work was supported by National Key R\&D Program of China (2019YFC1315800, 2019YFC1315802), National Natural Science Funds of China (No. 81830102; No. 81772578; No.81572367; No. 81772556), Shanghai Municipal Key Clinical Specialty, and Natural Science Funds of Shanghai (No. 21ZR1413800).

\section{Footnote}

Reporting Checklist: The authors have completed the STROBE reporting checklist. Available at http://dx.doi. org/10.21037/atm-20-8153

Data Sharing Statement: Available at http://dx.doi. org/10.21037/atm-20-8153

Conflicts of Interest: All authors have completed the ICMJE uniform disclosure form (available at http://dx.doi. org/10.21037/atm-20-8153). The authors have no conflicts of interest to declare.

Ethical Statement: The authors are accountable for all aspects of the work in ensuring that questions related to the accuracy or integrity of any part of the work are appropriately investigated and resolved. The study was conducted in accordance with the Declaration of Helsinki (as revised in 2013). Our Institutional Review Board approved the retrospective study (B2021-229). Due to the nature of retrospective study, no written informed consent was obtained from patients.

Open Access Statement: This is an Open Access article distributed in accordance with the Creative Commons Attribution-NonCommercial-NoDerivs 4.0 International License (CC BY-NC-ND 4.0), which permits the noncommercial replication and distribution of the article with the strict proviso that no changes or edits are made and the original work is properly cited (including links to both the formal publication through the relevant DOI and the license). See: https://creativecommons.org/licenses/by-nc-nd/4.0/.

\section{References}

1. Bray F, Ferlay J, Soerjomataram I, et al. Global cancer 
statistics 2018: GLOBOCAN estimates of incidence and mortality worldwide for 36 cancers in 185 countries. CA Cancer J Clin 2018;68:394-424.

2. Xu J, Fan J, Qin X, et al. Chinese guidelines for the diagnosis and comprehensive treatment of colorectal liver metastases (version 2018). J Cancer Res Clin Oncol 2019;145:725-36.

3. Chow FC, Chok KS. Colorectal liver metastases: An update on multidisciplinary approach. World J Hepatol 2019;11:150-72.

4. Brandi G, Corbelli J, de Rosa F, et al. Second surgery or chemotherapy for relapse after radical resection of colorectal cancer metastases. Langenbecks Arch Surg 2012;397:1069-77.

5. Imai K, Benitez CC, Allard MA, et al. Impact of Surgical Treatment for Recurrence After 2-Stage Hepatectomy for Colorectal Liver Metastases, on Patient Outcome. Ann Surg 2019;269:322-30.

6. van der Poel MJ, Barkhatov L, Fuks D, et al. Multicentre propensity score-matched study of laparoscopic versus open repeat liver resection for colorectal liver metastases. Br J Surg 2019;106:783-9.

7. Kanas GP, Taylor A, Primrose JN, et al. Survival after liver resection in metastatic colorectal cancer: review and meta-analysis of prognostic factors. Clin Epidemiol 2012;4:283-301.

8. Hur H, Ko YT, Min BS, et al. Comparative study of resection and radiofrequency ablation in the treatment of solitary colorectal liver metastases. Am J Surg 2009;197:728-36.

9. Lee WS, Yun SH, Chun HK, et al. Clinical outcomes of hepatic resection and radiofrequency ablation in patients with solitary colorectal liver metastasis. J Clin Gastroenterol 2008;42:945-9.

10. Benson AB 3rd, Venook AP, Cederquist L, et al. Colon Cancer, Version 1.2017, NCCN Clinical Practice Guidelines in Oncology. J Natl Compr Canc Netw 2017;15:370-98.

11. Jang HJ, Lim HK, Lee WJ, et al. Small hypoattenuating lesions in the liver on single-phase helical CT in preoperative patients with gastric and colorectal cancer: prevalence, significance, and differentiating features. J Comput Assist Tomogr 2002;26:718-24.

12. Lim GH, Koh DC, Cheong WK, et al. Natural history of small, "indeterminate" hepatic lesions in patients with colorectal cancer. Dis Colon Rectum 2009;52:1487-91.

13. Mohamed E, Adiamah A, Dunn WK, et al. Outcome of indeterminate liver lesions on computed tomography in patients with colorectal cancer. Ann R Coll Surg Engl 2018;100:382-7.

14. Schwartz LH, Gandras EJ, Colangelo SM, et al. Prevalence and importance of small hepatic lesions found at CT in patients with cancer. Radiology 1999;210:71-4.

15. Fukukura Y, Nakashima O, Kusaba A, et al. Angioarchitecture and blood circulation in focal nodular hyperplasia of the liver. J Hepatol 1998;29:470-5.

16. Nguyen BN, Fléjou JF, Terris B, et al. Focal nodular hyperplasia of the liver: a comprehensive pathologic study of 305 lesions and recognition of new histologic forms. Am J Surg Pathol 1999;23:1441-54.

17. Jones EC, Chezmar JL, Nelson RC, et al. The frequency and significance of small (less than or equal to $15 \mathrm{~mm}$ ) hepatic lesions detected by CT. AJR Am J Roentgenol 1992;158:535-9.

18. Wicherts DA, de Haas RJ, Sebagh M, et al. Regenerative nodular hyperplasia of the liver related to chemotherapy: impact on outcome of liver surgery for colorectal metastases. Ann Surg Oncol 2011;18:659-69.

19. Wiering B, Adang EM, van der Sijp JR, et al. Added value of positron emission tomography imaging in the surgical treatment of colorectal liver metastases. Nucl Med Commun 2010;31:938-44.

20. Parsai A, Miquel ME, Jan H, et al. Improving liver lesion characterisation using retrospective fusion of FDG PET/ CT and MRI. Clin Imaging 2019;55:23-8.

21. Friedrich-Rust M, Klopffleisch T, Nierhoff J, et al. Contrast-Enhanced Ultrasound for the differentiation of benign and malignant focal liver lesions: a meta-analysis. Liver Int 2013;33:739-55.

22. Trillaud H, Bruel JM, Valette PJ, et al. Characterization of focal liver lesions with SonoVue-enhanced sonography: international multicenter-study in comparison to CT and MRI. World J Gastroenterol 2009;15:3748-56.

23. Dietrich CF, Mertens JC, Braden B, et al. Contrastenhanced ultrasound of histologically proven liver hemangiomas. Hepatology 2007;45:1139-45.

24. Schwarze V, Marschner C, Negrão de Figueiredo G, et al. Single-Center Study: Evaluating the Diagnostic Performance and Safety of Contrast-Enhanced Ultrasound (CEUS) in Pregnant Women to Assess Hepatic Lesions. Ultraschall Med 2020;41:29-35.

25. Tang C, Fang K, Guo Y, et al. Safety of Sulfur Hexafluoride Microbubbles in Sonography of Abdominal and Superficial Organs: Retrospective Analysis of 30,222 Cases. J Ultrasound Med 2017;36:531-8.

26. Dietrich CF, Nolsøe CP, Barr RG, et al. Guidelines 
and Good Clinical Practice Recommendations for Contrast-Enhanced Ultrasound (CEUS) in the LiverUpdate 2020 WFUMB in Cooperation with EFSUMB, AFSUMB, AIUM, and FLAUS. Ultrasound Med Biol 2020;46:2579-604.

27. Crocetti D, Palmieri A, Pedullà G, et al. Peliosis hepatis: Personal experience and literature review. World J Gastroenterol 2015;21:13188-94.

28. Abdelli N, Bouhnik Y, Lavergne-Slove A, et al. Peliosis hepatitis during intestinal lymphomatous polyposis treated with chemotherapy and radiotherapy. Regression after antibiotic therapy. Gastroenterol Clin Biol 1995;19:537-41.

29. Simon DM, Krause R, Galambos JT. Peliosis hepatis in a patient with marasmus. Gastroenterology 1988;95:805-9.

30. Kim SH, Lee JM, Kim WH, et al. Focal peliosis hepatis as a mimicker of hepatic tumors: radiological-pathological correlation. J Comput Assist Tomogr 2007;31:79-85.

31. Yekeler E, Dursun M, Tunaci A, et al. Diagnosing of peliosis hepatis by magnetic resonance imaging. J Hepatol 2004;41:351.

32. Morris-Stiff G, White AD, Gomez D, et al. Nodular regenerative hyperplasia (NRH) complicating oxaliplatin chemotherapy in patients undergoing resection of colorectal liver metastases. Eur J Surg Oncol 2014;40:1016-20.

33. Rubbia-Brandt L, Lauwers GY, Wang H, et al. Sinusoidal obstruction syndrome and nodular regenerative hyperplasia are frequent oxaliplatin-associated liver lesions and partially prevented by bevacizumab in patients with hepatic colorectal metastasis. Histopathology 2010;56:430-9.

34. De Pasquale MD, Monti L, D'Andrea ML, et al. Focal nodular hyperplasia and hepatic regenerating nodules in pediatric oncology patients: how much invasive approach is necessary. Ann Hepatol 2013;12:308-14.

35. Donadon M, Di Tommaso L, Roncalli M, et al. Multiple focal nodular hyperplasias induced by oxaliplatin-based chemotherapy. World J Hepatol 2013;5:340-4.

36. Ünal E, Karaosmano lu AD, Ozmen MN, et al. Hepatobiliary phase liver MR imaging findings after Oxaliplatin-based chemotherapy in cancer patients. Abdom Radiol (NY) 2018;43:2321-8.

37. Arakawa Y, Shimada M, Utsunomya T, et al. Oxaliplatinrelated sinusoidal obstruction syndrome mimicking metastatic liver tumors. Hepatol Res 2013;43:685-9.

38. Shanbhogue AK, Virmani V, Vikram R, et al. Spectrum of medication-induced complications in the abdomen: role of cross-sectional imaging. AJR Am J Roentgenol 2011;197:W286-94.

39. Atila K, Coker A, Uçar D, et al. A rare clinical entity misdiagnosed as a tumor: peliosis hepatis. Ulus Travma Acil Cerrahi Derg 2007;13:149-53.

40. Goshima S, Kanematsu M, Kondo H, et al. Hepatic hemangioma: correlation of enhancement types with diffusion-weighted MR findings and apparent diffusion coefficients. Eur J Radiol 2009;70:325-30.

41. Yamashita Y, Ogata I, Urata J, et al. Cavernous hemangioma of the liver: pathologic correlation with dynamic CT findings. Radiology 1997;203:121-5.

42. Kim HD, Lim YS, Han S, et al. Evaluation of early-stage hepatocellular carcinoma by magnetic resonance imaging with gadoxetic acid detects additional lesions and increases overall survival. Gastroenterology 2015;148:1371-82.

43. Park MJ, Kim YK, Lee MW, et al. Small hepatocellular carcinomas: improved sensitivity by combining gadoxetic acid-enhanced and diffusion-weighted MR imaging patterns. Radiology 2012;264:761-70.

44. Wang WT, Zhu S, Ding Y, et al. T1 mapping on gadoxetic acid-enhanced MR imaging predicts recurrence of hepatocellular carcinoma after hepatectomy. Eur J Radiol 2018;103:25-31.

45. Morana G, Grazioli L, Kirchin MA, et al. Solid hypervascular liver lesions: accurate identification of true benign lesions on enhanced dynamic and hepatobiliary phase magnetic resonance imaging after gadobenate dimeglumine administration. Invest Radiol 2011;46:225-39.

46. Shin NY, Kim MJ, Lim JS, et al. Accuracy of gadoxetic acid-enhanced magnetic resonance imaging for the diagnosis of sinusoidal obstruction syndrome in patients with chemotherapy-treated colorectal liver metastases. Eur Radiol 2012;22:864-71.

47. Suh CH, Kim KW, Kim GY, et al. The diagnostic value of Gd-EOB-DTPA-MRI for the diagnosis of focal nodular hyperplasia: a systematic review and meta-analysis. Eur Radiol 2015;25:950-60.

48. Kim HJ, Lee SS, Byun JH, et al. Incremental value of liver MR imaging in patients with potentially curable colorectal hepatic metastasis detected at CT: a prospective comparison of diffusion-weighted imaging, gadoxetic acidenhanced MR imaging, and a combination of both MR techniques. Radiology 2015;274:712-22.

49. Niekel MC, Bipat S, Stoker J. Diagnostic imaging of colorectal liver metastases with CT, MR imaging, FDG PET, and/or FDG PET/CT: a meta-analysis of prospective studies including patients who have 
not previously undergone treatment. Radiology 2010;257:674-84.

50. Van Cutsem E, Cervantes A, Adam R, et al. ESMO consensus guidelines for the management of patients with metastatic colorectal cancer. Ann Oncol 2016;27:1386-422.
51. Zech CJ, Korpraphong P, Huppertz A, et al. Randomized multicentre trial of gadoxetic acid-enhanced MRI versus conventional MRI or CT in the staging of colorectal cancer liver metastases. Br J Surg 2014;101:613-21.

Cite this article as: Zhu K, Wang W, Luo R, Song D, Wang X, Gao Q, Fan J, Zhou J, Rao S, Wang X. Newly detected liver nodules with a history of colorectal cancer: are they metastatic? Review of 2,632 cases in a single center. Ann Transl Med 2021;9(13):1079. doi: 10.21037/atm-20-8153 